\title{
Engorde de bagres (Rhamdia quelen) en sistema de cultivo intensivo por sexos separados
}

\author{
Comolli, J.; Roux, J.P.; Sánchez, S.; Hernández, D.
}

Instituto de Ictiología, Facultad de Ciencias Veterinarias, Universidad Nacional del Nordeste, UNNE, Sargento Cabral 2139, Corrientes (3400), Argentina. E-mail: jacomolli@vet.unne.edu.ar.

\begin{abstract}
Resumen
Comolli, J.; Roux, J.P.; Sánchez, S.; Hernández, D.: Engorde de bagres (Rhamdia quelen) en sistema de cultivo intensivo por sexos separados. Rev. vet. 24: 2, 113-118, 2013. El bagre sudamericano (ramdiá o jundiá) se caracteriza por rápido crecimiento en los primeros meses de vida e importante diferencia de desarrollo entre ambos sexos. La madurez sexual del macho es precoz, por lo cual la hembra alcanza un peso vivo 30\% mayor que el del macho. El presente trabajo tuvo por objetivo analizar el engorde de especimenes de Rhamdia quelen separados por sexos en un sistema intensivo. Se llevaron a cabo tres tratamientos: TA- hembra, TB- mixto y TC- macho. El engorde se realizó en cajas plásticas de 30 litros de capacidad. Se utilizaron 81 ejemplares, cuyos pesos medios fueron de 49,30 y 42,90 g para hembras y machos respectivamente. Los peces se distribuyeron a razón de 9 ejemplares por caja, con una densidad de 300 individuos $/ \mathrm{m}^{3}$. El ensayo tuvo una duración de 60 días. Las variables analizadas fueron: coeficiente de crecimiento específico, consumo diario, tasa de conversión alimenticia, tasa de supervivencia, biomasa total producida, porcentaje de cabeza, rendimiento de carcasa (RC) e índice gonadosomático (IGS). No se observaron diferencias significativas entre los tratamientos para las variables estudiadas ( $>0,05)$, pero cuando se compararon las variables de las hembras se encontró que RC e IGS resultaron significativamente diferentes entre los tratamientos A y B, con valores medios de 89,47 y $2,35 \%$ frente a 88,01 y $3,79 \%$, respectivamente. Los valores de RC fueron similares a los reportados por otros autores, pero los de IGS fueron menores, en particular para las hembras. Las hembras TA tuvieron valores más bajos de IGS y más altos de RC que las hembras TB. Estos resultados indicarían que el engorde de $R$. quelen por sexos separados produce mejor rendimiento de carcasa en las hembras debido al menor índice gonadosomático. En contraste, no se encontraron estos cambios en los machos.
\end{abstract}

Palabras clave: Rhamdia quelen, bagre sudamericano, nutrición, rendimiento de carcasa, índice gonadosomático.

\begin{abstract}
Comolli, J.; Roux, J.P.; Sánchez, S.; Hernández, D.: Fattening of ramdiá (Rhamdia quelen) in intensive culture system by separated sexes. Rev. vet. 24: 2, 113-118, 2013. The South American catfish (ramdiá or jundiá) is characterized by a fast growth during the first months of life, and an important difference of development between sexes. Female reaches $30 \%$ more living weight than males, because the latter become sexually mature faster. The aim of this study was to analyze the fattening of Rhamdia quelen by separated sexes in an intensive system. Three treatments were made: TA- female, TB- mix and TC- male. Fattening was carried out in 301 plastics boxes. Eighty one specimens were used, with an average weight of $49.30 \mathrm{~g}$ for female and $42.90 \mathrm{~g}$ for male. Nine fishes per box were distributed at a density of 300 fishes $/ \mathrm{m}^{3}$. The essay lasted 60 days. Analyzed variables were: specific growth coefficient, daily food intake, feed conversion rate, survival rate, total biomass produced, head percentage, carcass performance (RC), and gonadosomatic index (IGS). No significant differences were found among treatments for the studied variables ( $>0.05)$. By comparing the variables between sexes, it was found that RC and IGS from TA had significant differences compared to TB, with average values of 89.47 and $2.35 \%$ versus 88.01 and $3.79 \%$ respectively. RC values were similar to those of other authors, but IGS were lower, particularly in females. Females from TA had lower values of IGS and better performance values of RC than the females from TB. These results may suggest that better RC values due to lower IGS can be obtained when females and males are grown separately. Physical sex separation of $R$.
\end{abstract}


quelen during the fattening stage produces a better carcass performance in females because of their decreased gonadosomatic index. In contrast, these changes were not found in males.

Key words: Rhamdia quelen, South American catfish, nutrition, carcass performance, gonadosomatic index.

\section{INTRODUCCIÓN}

El bagre sudamericano tiene una gran adaptación a la cría en cautiverio ${ }^{3}$. En la naturaleza el macho crece más rápidamente que la hembra hasta el primer o segundo año de vida, momento en el cual la hembra llega a tener un crecimiento mayor ${ }^{12}$. Asimismo, en cultivo se observa que las hembras crecen hasta $30 \%$ más que los machos, con pesos de 900 a $1000 \mathrm{~g}$ para las hembras y de 600 a $800 \mathrm{~g}$ para los machos, aproximadamente a los seis meses ${ }^{1,27}$. Esta situación podría deberse a una más precoz maduración sexual de los machos ${ }^{14}$.

El período reproductivo varía de acuerdo a la región geográfica: en cercanía de los trópicos se extiende desde agosto a abril y se reduce al alejarse de dichas zonas ${ }^{23}$. El desove de Rhamdia quelen es asincrónico ${ }^{12}$. Esta especie no presentaría un período reproductivo muy marcado, ya que los desoves se producen a medida que las ondas ovocitarias maduran ${ }^{2,30}$. El marcado dimorfismo sexual que aparece en algunas especies al alcanzar la madurez reproductiva, o el cese de la alimentación durante el período reproductivo, denotarían posibles influencias del status hormonal, en este caso de las hormonas sexuales, sobre el desencadenamiento del comportamiento alimentario ${ }^{8}$.

Una de las características del crecimiento de este bagre es el mayor desarrollo de la hembra, determinando que las prácticas de manejo se orienten a explotar esta particularidad por medio de la reversión sexual ${ }^{29}$. Otra posibilidad es el manejo monosexo, dado que esta especie presenta cierto dimorfismo sexual permitiendo la diferenciación entre machos y hembras al alcanzar la edad reproductiva ${ }^{1,22,27}$. Aún no se han realizado trabajos que exploren esta posibilidad; quizás realizando una selección por sexo como práctica de manejo para la etapa de engorde, se lograrían mejores niveles de producción ${ }^{1,9}$. El presente trabajo tuvo por objetivo analizar el engorde de machos y hembras de $R$. quelen juntos o separados bajo un sistema intensivo de cultivo.

\section{MATERIAL Y MÉTODOS}

El trabajo fue realizado en las instalaciones del Instituto de Ictiología mencionado ut supra (INICNE). Se utilizaron 81 ejemplares de $R$. quelen de un año de vida, mantenidos en retención (crecimiento diferido) durante este período, con signos externos de madurez sexual y peso medio de $49,28 \pm 25,15$ y $42,88 \pm 20,32 \mathrm{~g}$ para hembras y machos, respectivamente. Los peces fueron distribuidos en 9 cajas plásticas de 30 litros de capacidad, a razón de 9 individuos por caja, a una densidad estimada de 300 ejemplares $/ \mathrm{m}^{3}$. Se empleó un diseño experimental completamente aleatorizado que incluyó 3 tratamientos y 3 repeticiones: TA- hembra, TB- mixto y TC- macho $(n=9)$. El sexado se realizó por observación directa (Figura 1 a y b).

La experiencia tuvo una duración de 60 días, período durante el cual los peces fueron alimentados con ración balanceada comercial extrusada con un contenido de $28,2 \%$ de proteína bruta, $12,7 \%$ de extracto etéreo, $32,9 \%$ de hidratos de carbono (determinaciones realizadas en laboratorios del INICNE ) y $3134,6 \mathrm{kcal} /$ $\mathrm{kg}$ de energía digestible, la cual fue estimada según el método de Jobling ${ }^{18}$. La ración se suministró diariamente ad libitum a las 8:00 h y a las $16: 00 \mathrm{~h}$ se procedió al retiro del alimento sobrante. Durante el período experimental, en cada una de las cajas se determinaron los parámetros básicos de calidad de agua: temperatura, $\mathrm{pH}$, conductividad y oxígeno disuelto (\% y $\mathrm{mg} / \mathrm{l})$.

Al finalizar el ensayo los peces fueron pesados y contados para calcular los siguientes parámetros: biomasa producida $(\mathrm{BT})=$ peso final - peso inicial; coeficiente de crecimiento específico $(\mathrm{CCE})=[3$ (peso fi$\mathrm{nal}^{0,33}$ - peso inicial $\left.\left.{ }^{0,33}\right) \times 100\right] /$ tiempo en días; tasa de supervivencia $(\mathrm{TS})=\left(\mathrm{N}^{o}\right.$ peces final x 100) $/ \mathrm{N}^{\circ}$ peces iniciales; ganancia diaria de peso $(\mathrm{GDP})=\mathrm{BT} /$ días; consumo diario $(\mathrm{CD})=$ consumo total $/[$ [peso final peso inicial) / 2] / días x 100 y tasa de conversión alimentaria $(\mathrm{TCA})=$ ración consumida $/ \mathrm{BT}$.

Los parámetros de rendimiento de faena fueron: rendimiento de carcasa $(\mathrm{RC})=100(\mathrm{PE} / \mathrm{PV})$, donde $\mathrm{PE}$ es el peso de los individuos eviscerados (se considera la carcasa con cabeza y branquias) y $\mathrm{PV}$ es peso vivo; porcentaje de cabeza $(\mathrm{PC})=100$ (peso cabeza/ PV) e indice gonadosomático $(\mathrm{IGS})=100$ (peso gonadal/ PV).

Los datos fueron procesados estadísticamente empleando el programa Infostat $1.1^{16}$. Los resultados obtenidos para los tres tratamientos se evaluaron mediante ANOVA a una vía para cada una de las variables analizadas y cuando se detectaron diferencias significativas, las comparaciones a posteriori se realizaron mediante el test de Tukey $(\mathrm{p}<0,05)$. Adicionalmente, IGS, PC y RC fueron comparados entre los sexos (TA vs hembras de TB y TC vs machos de TB), debido a que estas variables están influenciadas por desarrollo diferencial de las gónadas entre los sexos.

\section{RESULTADOS Y DISCUSIÓN}

Los valores medios obtenidos para las variables de calidad de agua fueron $26,35^{\circ} \mathrm{C} ; 6,57 ; 94,29 \mu \mathrm{S} / \mathrm{cm}$ y $6,06 \mathrm{mg} / \mathrm{l}$. Durante el período experimental no se ob- 
servaron diferencias significativas $(\mathrm{p}>0,05)$ de los parámetros de calidad de agua entre los diferentes tratamientos.

Crecimiento, consumo de alimento y supervivencia. Al final de la experiencia la TS no registró diferencias significativas entre tratamientos $(p>0,05)$. Las medias por tratamientos fueron de $81,48 \%, 85,19 \%$ y $92,59 \%$ para TA, TC y TB, respectivamente. La TS obtenida resultó inferior a la reportada en estudios realizados en estanques, jaulas y cajas de recirculación (entre 95 y 100\%), lo cual podría relacionarse con una mejora del bienestar general de los peces en relación al sistema aquí empleado ${ }^{19}$. Se corroboró un $100 \%$ de efectividad del sexado manual realizado previamente a la formación de los grupos experimentales (Figura 1 a y b).

Los valores de GDP, CCE, CD y TCA no arrojaron diferencias significativas $(p>0,05)$ entre los tratamientos (Tabla 1). En todas las cajas se observó una buena aceptación del alimento balanceado. Valores de CCE cercanos al $8 \%$ fueron obtenidos en ejemplares de $R$. quelen retenidos durante un año, que posteriormente fueron engordados en estanques a diferentes densidades $\left(1,2\right.$ y 3 ejemplares $\left./ \mathrm{m}^{2}\right)$, existiendo una relación negativa entre densidad de siembra y $\mathrm{CCE}^{28}$. En estudios realizados en esta especie en jaulas con densidades de 250 individuos $/ \mathrm{m}^{3}$ se obtuvieron GPD de 1,50 a 1,90 $\mathrm{g} /$ día $^{21}$, valores muy superiores a los obtenidos en el presente trabajo. Sin embargo, se obtuvieron GDP de
0,21 a 0,28 g/día en el engorde de ejemplares juveniles en cajas de 50 litros, a densidades de 500 peces $/ \mathrm{m}^{3}$ y alimentados con una ración conteniendo $28 \%$ de proteínas ${ }^{13}$, siendo estos valores muy cercanos a los obtenidos en el presente trabajo.

El CD del alimento fue inferior al comunicado por otros autores ${ }^{18}$. El CD no aumentó, hecho esperado por efecto del crecimiento compensatorio para animales que se encontraban en retención ${ }^{15,17}$. Las TCA estimadas resultaron muy elevadas para el engorde de esta especie, pues según estudios previos se ubicarían entre 1,3 y $3,1^{18}$.

La cría de ejemplares juveniles de $R$. quelen en jaulas flotantes produce un efecto estresante en esta categoría de animales, generando mayores niveles de cortisolemia que los registrados en peces mantenidos en estanques, evidenciando una situación de estrés crónico ${ }^{5}$. Lo mencionado podría explicar los bajos rendimientos del presente estudio.

Todos los parámetros evaluados fueron inferiores a lo esperado en este sistema de producción. En este contexto, una insuficiente adaptación al sistema pudo haber ocurrido a pesar del período previo de aclimatación a las condiciones experimentales al que se sometió a los peces. La falta de adaptación podría haber derivado en una mala nutrición, pues el estrés conduciría al desbalance energético, exigiendo la utilización de las proteínas dietarias para contrarrestar dicha situación. Ello permitiría explicar, al menos en parte, la elevada relación de la TCA.
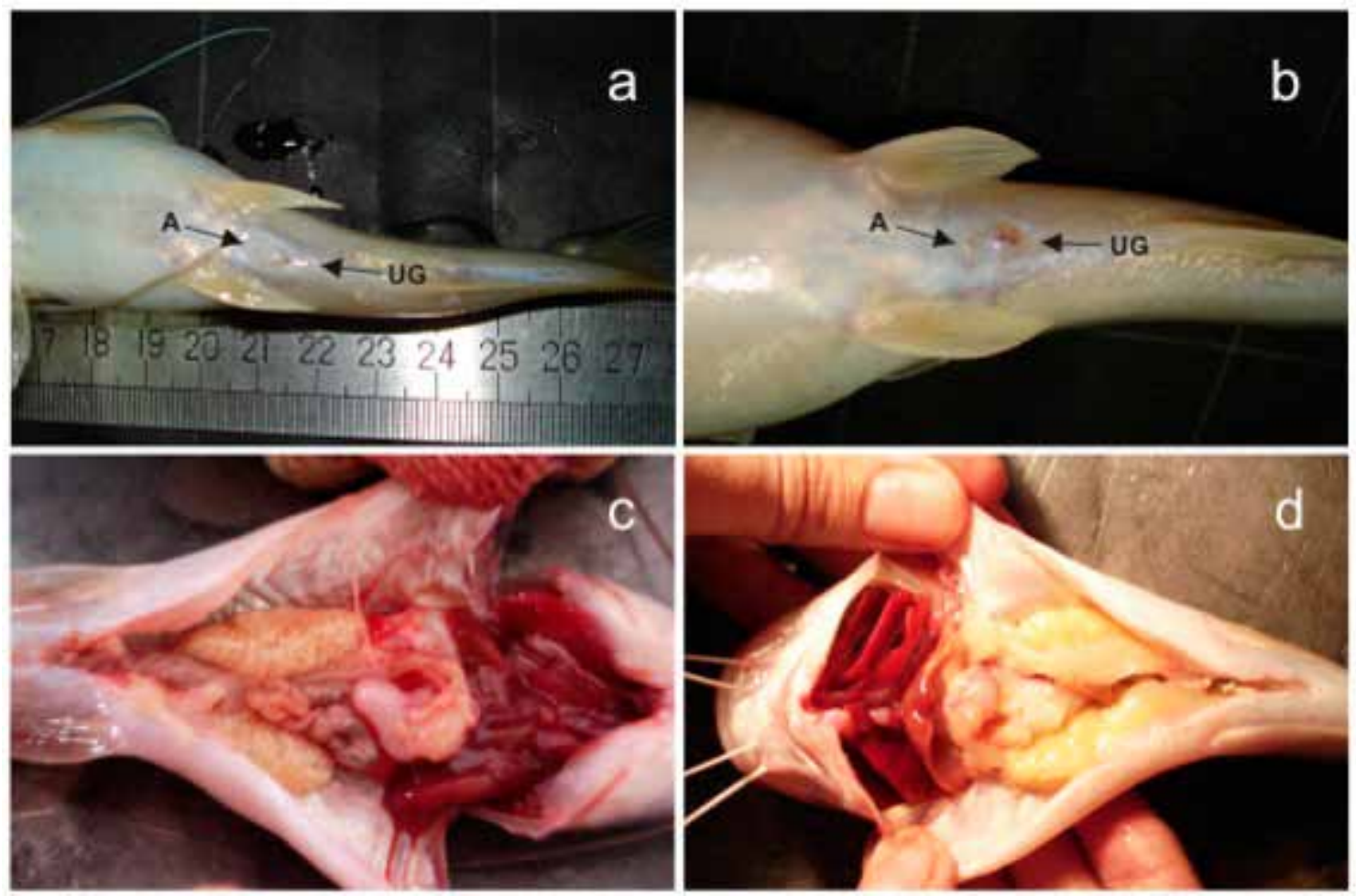

Figura 1. (a-b): características morfológicas del poro genital en reproductores de R. quelen. (a): macho, orificio anal (A) y abertura urogenital (UG) donde se puede observar la presencia de líquido seminal (espermiación). (b): hembra, orificio anal (A) y abertura urogenital (UG). (c-d): observación del estado de actividad gonadal en hembras correspondientes a los tratamientos B (c) y A (d). 
Tabla 1. Resultados del engorde y rendimientos a la faena, según tratamiento.

\begin{tabular}{ccccccc}
\hline $\mathrm{t}$ & CCE (\%) & GDP g/día & CD (\%) & TCA (g) & RC (\%) & PC (\%) \\
\hline TA & 0,77 & 0,12 & 0,87 & 4,17 & 89,47 & 19,99 \\
TB & 0,72 & 0,09 & 0,85 & 4,19 & 88,68 & 19,38 \\
TC & 0,55 & 0,07 & 1,01 & 6,56 & 89,66 & 18,79 \\
\hline
\end{tabular}

$\mathrm{t}$ : tratamientos: TA-hembras, TB-mixto, TC-machos, CCE: coeficiente de crecimiento específico, GDP: ganancia diaria de peso, $\mathrm{CD}$ : consumo diario, TCA: tasa de conversión alimenticia, RC: rendimiento de carcasa, PC: porcentaje de cabeza.

Tabla 2. Rendimientos a la faena, según sexos y tratamiento.

\begin{tabular}{lcccc}
\hline sexo & $\mathrm{t}$ & $\mathrm{RC}(\%)$ & PC (\%) & IGS (\%) \\
\hline \multirow{2}{*}{ machos } & TA & $89,66^{\mathrm{a}}$ & $18,79^{\mathrm{a}}$ & $2,46^{\mathrm{a}}$ \\
& TC & $89,41^{\mathrm{a}}$ & $19,11^{\mathrm{a}}$ & $2,58^{\mathrm{a}}$ \\
\hline \multirow{2}{*}{ hembras } & TB & $89,47^{\mathrm{a}}$ & $19,99^{\mathrm{a}}$ & $2,35^{\mathrm{a}}$ \\
& TC & $88,01^{\mathrm{b}}$ & $19,62^{\mathrm{a}}$ & $3,79^{\mathrm{b}}$ \\
\hline
\end{tabular}

$\mathrm{t}$ : tratamientos: TA-hembras, TB-mixto, TC-machos, RC: rendimiento de carcasa, PC: porcentaje de cabeza, IGS: índice gonadosomático. Letras diferentes representan diferencias significativas entre tratamientos $(p<0,05)$.

Tabla 3. Índices gonadosomáticos obtenidos por otros autores en distintas estaciones del año.

\begin{tabular}{lccc}
\hline autores & estación & IGS $(\%)$ & observaciones \\
\hline Carneiro et al. $(2004)^{6}$ & ene-feb & $4,53-11,11$ & - \\
Ghiraldelli et al. $(2007)^{11}$ & mayo & 8,5 & rango de 0,11-13,03\% \\
Reidel et al. $(2010)^{25}$ & sept-dic & $10-15$ & en otoño varió entre 2 y 5\% \\
Felin et al. $(2007)^{9}$ & marzo & 9 & - \\
Barcellos et al. $(2001)^{3}$ & mar-abr & 8 & segunda maduración gonadal \\
\hline
\end{tabular}

Rendimientos de faena. Los valores de RC y PC no asumieron diferencias significativas entre tratamientos ( $\mathrm{p}>0,05)$ (Tablas 1 y 2). RC fue similar para los dos sexos con valores cercanos al $90 \%$, tanto en ejemplares criados conjunta como separadamente. Valores inferiores de $\mathrm{RC}$, entre 80 a $82 \%$, fueron obtenidos por otros autores, uno de ellos trabajando con ejemplares juveniles de $R$. quelen alimentados con diferentes fuentes lipídicas durante 26 días ${ }^{23}$, y el otro estudiando distintas fuentes proteicas para el engorde de $R$. quelen ${ }^{18}$. Contrariamente, se reportaron valores de RC superiores al $85 \%{ }^{6,7}$, en concordancia con los resultados obtenidos en el presente trabajo.

En otros ensayos se registraron diferencias entre machos y hembras, que fueron atribuidas al peso gonadal de las hembras y correspondieron al 11,11\% del peso vivo ${ }^{6}$. Se afirma que el RC de las hembras varía de acuerdo a la época del año, en relación a la madurez sexual ${ }^{25}$. Los animales de mayor tamaño revelan una RC significativamente menor a la de los individuos de menor peso ${ }^{24}$.
En cuanto al PC, se reportan valores de $20 \%$ en especimenes juveniles de Rhamdia $s p^{24}$, similares a los del presente trabajo. Sin embargo, se han obtenido niveles de $16 \%$ con dietas conteniendo $25 \%$ de proteínas, los cuales disminuyeron al aumentar la tasa proteica alimentaria de 30 a $35 \%{ }^{26}$. Estos resultados demuestran que el PC es influenciado por el nivel de proteínas de la dieta, así como también por el desarrollo del animal: cuanto más grande es el pez, su cabeza es más pequeña en relación a su cuerpo por lo cual el PC disminuye, no habiendo relación aparente con el sexo del animal.

Cuando se compararon los resultados para cada sexo, en el caso de los machos no surgieron diferencias (Tabla 2). En cambio, se registraron diferencias significativas $(\mathrm{p}<0,05)$ de RC e IGS entre hembras de los tratamientos A y B, donde la hembras del tratamiento monosexo tuvieron mayor RC y menor IGS que las hembras del tratamiento mixto (Tabla 2). Como se vio, el período reproductivo de $R$. quelen abarca desde agosto a marzo ${ }^{1}$. Esto, sumado a la característica asincrónica del desove de esta especie ${ }^{1,11,12}$, debe ser tenido en cuenta para determinar el estado reproductivo de los animales estudiados. Una de las características que confirmó que los ejemplares estudiados se encontraban en actividad reproductiva fue el hecho de que el $100 \%$ de los machos estuvieron en estado de espermiación (Figura 1a).

En otras investigaciones los IGS de los machos variaron acorde a la época del año, reportándose valores de 3,4\% para los meses de marzo-abril ${ }^{4}$, de $2,73 \%$ en machos criados en estanque en el mes de marzo ${ }^{10} \mathrm{y}$ de 3,47 a $4,28 \%$ en animales de 11 meses de edad ${ }^{6}$. Por otro lado, experiencias realizadas en Brasil mostraron picos reproductivos que ocurrieron en noviembre-diciembre, con IGS de 4,73 a 7,32 ${ }^{20}$. Los valores medios de IGS para peces maduros es de 3,5 a $4 \%$, aunque pueden llegar hasta $9,16 \%{ }^{11}$. En el presente trabajo los machos se comportaron en general como los citados en las referencias bibliográficas teniendo en cuenta la época del año, destacando que se registraron machos en espermiación con IGS tan bajos como 2,46.

Los IGS de machos y hembras no exhibieron diferencias significativas. En otras experiencias el IGS de $R$. quelen comenzó a aumentar en abril, alcanzando en septiembre una gran diferencia entre sexos, que se acentuó aún más hacia el mes de diciembre ${ }^{11,26}$. Algunos investigadores hallaron diferencias significativas del IGS entre machos y hembras $(2,6$ y $9 \%$, respectivamente) ya en el mes de marzo, al finalizar el período reproductivo ${ }^{9}$.

Entre las hembras TA y TB se encontraron diferencias significativas para RC e IGS, con un mejor RC y menor IGS en las hembras cultivadas individualmente que en aquéllas que compartieron el ambiente con los machos (Figura 1 c y d). Al analizar los IGS reportados 
por otros autores se aprecia que en general los resultados aquí obtenidos se encuentran por debajo de lo esperado (Tabla 3).

De los diferentes parámetros evaluados, solo se hallaron diferencias significativas en RC e IGS en las hembras cultivadas individualmente respecto de aquéllas que se mantuvieron junto a los machos. Los resultados indicarían que al cultivarse ambos sexos juntos se mantendría un estímulo sexual que determinaría que las hembras se mantengan activas, dirigiendo parte de la energía disponible hacia las gónadas en detrimento de otros procesos fisiológicos. Esto llevaría a un menor $\mathrm{RC}$, situación que fue observada en este trabajo (Tabla 2).

En conclusión, la separación de sexos en la etapa de engorde del bagre sudamericana ( $R$. quelen) produce mejores rendimiento de carcasa en las hembras, debido a una disminución del índice gonadosomático, cambio que no ocurre en los machos.

\section{REFERENCIAS}

1. Baldisserotto B, Radünz J. 2004. Criação de jundiá, Ed. UFSM, Santa Maria (Brasil), 232 p.

2. Baldisserotto B. 2009. Fisiologia de peixes aplicada à piscicultura, $2^{\circ}$ ed., UFSM. Santa Maria (Brasil), $352 \mathrm{p}$.

3. Barcellos LJ, Woehl VM, Wassermann GF, Krieger MH, Quevedo RM, Lulhier F. 2001. Plasma levels of cortisol and glucose in response to capture and tank transference in Rhamdia quelen (Quoy and Gaimard), a South American catfish. Aquac Res 32: 123-125.

4. Barcellos LJ, Wassermann G, Scott A, Woehl V, Quevedo R, Ittsés I, Krieger M, Lulhier F. 2002. Plasma steroid concentrations in relation to the reproductive cycle of cultured male Rhamdia quelen. J Fish Biol 61: 751-763.

5. Barcellos L, Kreutz L, Quevedo R, Fioreze I, Cericato L, Soso A, Fagundes M, Conrad J, Baldissera R, Bruschi A, Ritter F. 2004. Nursery rearing of jundia, Rhamdia quelen (Quoy \& Gaimard) in cages: cage type, stocking density and stress response to confinement. Aquaculture 232: 383-394.

6. Carneiro P, Mikos J, Bendhack F, Ignácio S. 2004. Processamento do jundiá Rhamdia quelen: rendimento de carcaça. Rev Acad Ciênc Agrár Ambient 2: 11-17.

7. Corrêia V, Radünz J, Lazzari R, Veiverberg C, Bergamin G, Pedron F, Ferreira C, Emanuelli T, Ribeiro C. 2009. Crescimento de jundiá e carpa húngara criados em sistema de recirculação de água. Ciênc Rural 39: 1533-1539.

8. Cuenca E, García Gallardo M. 1987. Ingesta y conducta alimentaria. En: Nutrición en Acuicultura (Espinosa J, Labarta U, ed.), Ed. CAICYT, Madrid, 303 p.

9. Felin R, Coldebella JI, Manfio ML. 2007. Avaliação de machos e fêmeas de Rhamdia quelen criados em tanques-rede de pequeno volume. Anais Workshop sobre Jundiá, Santa Maria, RS (Brasil), p. 41.

10. Fracalossi D, Meyer G, Santamaria F, Weingartner M, Zaniboni E. 2004. Desempenho do jundiá, Rhamdia quelen, e do dourado, Salminus brasiliensis, em viveiros de terra na região sul do Brasil. Acta Sci Anim Sci 26: 345-352.
11. Ghiraldelli L, Machado C, Fracalossi D, Zaniboni E. 2007. Desenvolvimento gonadal do jundiá, Rhamdia quelen (Teleostei, Siluriformes) em viveiros de terra, na região sul do Brasil. Acta Sci Biol Sci 29: 349-356.

12. Gomes LC, Golombieski JI, Chippari AR, Baldisserotto B. 2000. Biologia do Jundiá (Teleostei, Pimelodidae). Ciênc Rural 30: 179-185.

13. Graeff A, Tomazelli A, Pruner E. 2006. Desenvolvimento corporal de jundiás (Rhamdia quelen) alimentados com dietas completas contendo diferentes níveis de energia na fase de engorda. Anales IV Congreso Iberoamericano Virtual de Acuicultura, Zaragoza, España, p. 56-61.

14. Graeff A, Tomazelli A, Pruner EN. 2008. Variação percentual e freqüencia de alimento fornecido no desenvolvimento final de jundiás (Rhamdia quelen) na fase de póslarvas. Rev Electrón Vet 9: 1-9.

15. Hepher B. 1988. Nutrición de peces comerciales en estanques, Limusa, México, $406 \mathrm{p}$.

16. InfoStat. 2002. InfoStat, versión 1.1. Grupo InfoStat, FCA, Universidad Nacional de Córdoba, Argentina.

17. Jobling M. 1994. Fish bioenergetics, Chapman and Hall, London, $294 \mathrm{p}$.

18. Jobling M. 1995. Environmental biology of fishes, Chapman and Hall, New York, 455 p.

19. Lazzari R, Radünz J, Emanuelli T, Araújo F, Leão M, Losekann M, Correia V, Bochi V. 2006. Diferentes fontes protéicas para a alimentação do jundiá (Rhamdia quelen). Ciênc Rural 36: 240-246.

20. Lucélia T, Pereira C, Rebechi T, Giovano N, Andrei RK, Fábio M, Marçal MR, Robie AB. 2011. Growth and reproductive characteristics of Rhamdia quelen males fed on different digestible energy levels in the reproductive phase. Aquaculture 326: 74-80.

21. Luchini L, Quiros R. 1990. Cage culture of south american catfish (Rhamdia sapo). Preliminary results in the Salto Grande reservoir (Argentina). J Aquac Trop 5: 163-172.

22. Mardini CV, Silveira MA, Barenho DH. 1981. Técnica de indução da desova em jundiá (Rhamdia quelen) empregada na estação experimental de piscicultura da Lagoa dos Quadros, Ed. Secretaria da Agricultura, Porto Alegre (Brasil), 14 p.

23. Melo J, Radünz J, Souza J, Trombetta C. 2002. Desenvolvimento e composição corporal de alevinos de jundiá (Rhamdia quelen) alimentados com dietas contendo diferentes fontes de lipídios. Ciênc Rural 32: 323-327.

24. Pouey J, Miotto H, Kunz T. 1999. Principais componentes corporais do jundiá Rhamdia sp cultivado na densidade de um peixe $/ \mathrm{m}^{2}$ e dividido em quatro faixas de peso. Anais Reunião Anual da Sociedade Brasileira de Zootecnia, Porto Alegre, RS (Brasil), p. 36.

25. Reidel A, Boscolo W, Feiden A, Romagosa E. 2010. The effect of diets with different levels of protein and energy on the process of final maturation of the gametes of Rhamdia quelen stocked in cages. Aquaculture 298: 354-359.

26. Reidel A, Romagosa E, Feiden A, Boscolo W, Coldebella A, Signor A. 2010. Rendimento corporal e composição química de jundiás alimentados com diferentes níveis de proteína e energia na dieta, criados em tanques-rede. Rev Bras Zootecn 39: 233-240. 
27. Rossi F, Luchini L. 2008 Cultivo de randiá (Rhamdia quelen) para fomento de su producción comercial, en clima templado-cálido. En: Desarrollo de tecnologías para producción del randiá (Rhamdia quelen), Ed. SAGPYA, Serie Pesca y Acuicultura No 2, Buenos Aires, 67 p.

28. Sánchez S, Santinón J, Hernández D, Roux J, Domitrovic H. 2008. Cría de bagre sudamericano (Rhamdia quelen) en estanques luego de diferentes períodos de retención del crecimiento a tres densidades de siembra. Rev Electrón Vet 9: 1-9.
29. Soller F, Moreira R, Orozco C, Hilsdorf A. 2007. Triploidy induction by cold shock in the South American catfish, Rhamdia quelen (Siluriformes, Quoy and Gaimard, 1824). Aquaculture 272: 110-114.

30. Vazzoler AE. 1996. Biologia da reprodução de peixes teleósteos: teoria e prática, Ed. EDUEM, Maringá, SP (Brasil), $169 \mathrm{p}$.

\section{Asociación Cooperadora de la Facultad de Ciencias Veterinarias Universidad Nacional del Nordeste}

Personería Jurídica No 647/92 y 912/00

Sargento Cabral 2139

3400 Corrientes

Argentina

La Asociación Cooperadora de la Facultad de Ciencias Veterinarias de la UNNE fue constituida el 10 de diciembre del año 1991 como entidad de bien público, con el objeto de promover y coadyuvar las actividades científicas, educativas y culturales relacionadas con las ciencias veterinarias. En tal sentido, implementa acciones para colaborar con la enseñanza, extensión, actualización y difusión científica que realiza dicha casa de estudios.

\section{Beneficios que brinda a sus asociados:}

- Fotocopias con descuentos especiales del $20 \%$ en la Fotocopiadora Copias.com que funciona dentro del predio de la Facultad de Ciencias Veterinarias.

- Descuentos del 10\% para la adquisición de libros de la Editorial Inter-Médica.

- Descuentos especiales en otros rubros.

Sea solidario, asóciese en beneficio de toda la comunidad universitaria

Tel. 03783-425753 interno 186•Página Web www.vet.unne.edu.ar•E-mail: cooperadora@vet.unne.edu.ar 